\title{
The Problem of Wave Diffraction in Elastic Medium is Solved using the Integral Averaged Differential Method
}

\author{
Phan Huy Thien ${ }^{1}$, Phan Van Hap ${ }^{2}$ \\ Department of Theoretical Physics, Faculty of Physics, Hanoi University of Science, \\ Vietnam National University \\ 334 Nguyen Trai street, Thanh Xuan, Hanoi, Vietnam
}

\begin{abstract}
Wave diffraction in an elastic medium with cavity with hard wall is a physic problem with many potential applications in physics, in fluid dynamics, water irrigation. The boundary problem is transformed into a system of integral equation with singularity. After that, the integral averaged differential (IAD) method is used to calculate the integral and solve the equation. Our results are given for the case the opening angle is $p / 2$ and $p / 3$. Comparision to the results of Refences [4] shows the advantage of IAD method.
\end{abstract}

Keywords: wave diffraction of elastic medium with gaping corner, integral equations singularity.

\section{Introduction}

The problem of wave diffraction in an elastic media with an opening angle is considered based on the wave equations with different hard wall boundary. Different methods have been proposed to olve this problem such as the partial differential equation method, finite difference method, The case, the opening angle $b=0$ has already solved. For the opening angle $0<b<p$ has many potential applications in physics such as explorative detonations, designs for water dam, ocean dam, water irrigation, and are actively studied. Method of solution using integral equation system with singularity is a new direction for these applied physics problem. It is based on the integral averaged differential method proposed by Phan Van Hap [5, 9]

Let us start from the equation:

$$
r \frac{\mathbb{q}^{2} \mathbf{u}}{\mathbf{q} t^{2}}=(l+2 m) \text { grad } \operatorname{div} \mathbf{u}-\operatorname{rot} \operatorname{rot} \mathbf{u}
$$

Where $\mathbf{u}$ is the displacement vector; $l, m$ are elastic constants, $r$ is the mass density of the medium.

Express vector $\mathbf{u}$ through the scalar potential $j$ and the vector potential $y$ we obtain:

$$
\mathbf{u}=\text { gradj }+\operatorname{rot} \psi \text { with } \operatorname{div} \psi=0
$$

Substitute (2) into (1) we get:

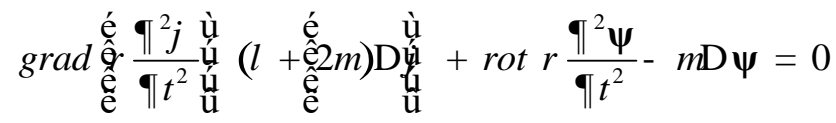

From this:

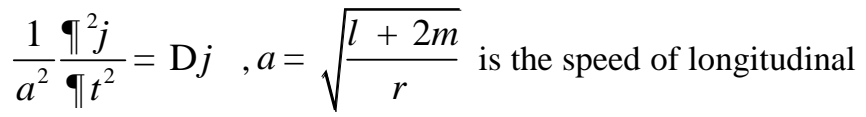
wave,

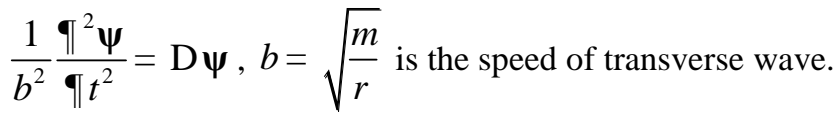

Satisfy (3). For a planar geometry, $\left(u_{z}=0\right)$, we have:

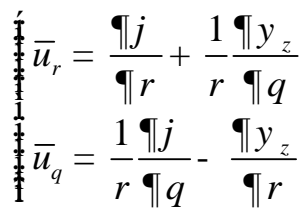

This leads to the equations:

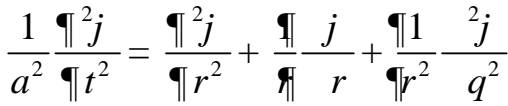

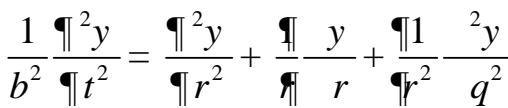

In the region $0 £ ₹ ¥-£ £ a \quad q \quad a \quad(b=2 a)$ a planar wave is propagated:

$$
j_{0}=f \text { '́at - } r \cos \left(q-a-e_{1}\right) \grave{c}
$$

where $f$ is a known function of real argument; a is speed of wave propagation. The wave front (5) at time $t=0$ goes through the origin, and for $t>0$ is the $N M M \nsubseteq \notin$ in Fig. 1.

Suppose the longitudinal wave speed is a, transverse wave speed is b. the system of equation (4) has the boundary condition

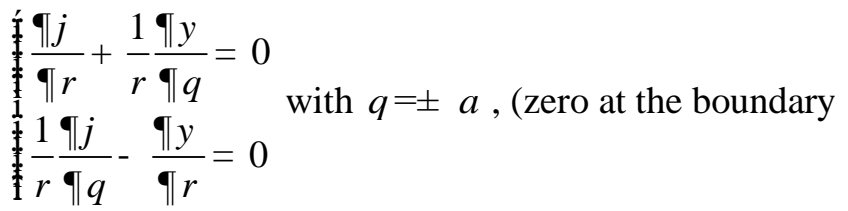

Consider for hard wall) $(*)$

$$
f(x)=\frac{\begin{array}{l}
1 \text { with } x^{3} \quad 0 \\
1
\end{array} 0 \text { with } x<0}{1}
$$

We consider the class of solution:

$$
u=\operatorname{Re} e_{E}(x+i y) \text { ù }
$$

For example:

$$
j=j\left(\mathrm{~W}_{1}\right)
$$

where $\mathrm{W}_{1}=\mathrm{W}_{1}(r, j, t)$ and $j$ are arbitrary analytic function. Substitute into Eq. (4) we get: 


\section{International Journal of Science and Research (IJSR) \\ ISSN (Online): 2319-7064}

Index Copernicus Value (2013): 6.14 | Impact Factor (2014): 5.611

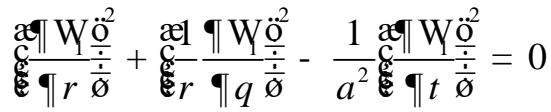

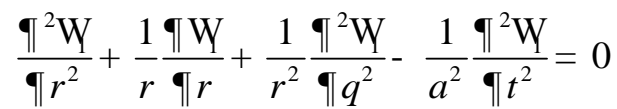

It is easily seen that this system of equation is satisfied for $\mathrm{W}_{1}=q \pm c_{1}, c_{1}=\arccos \frac{a t}{r}$.

Thus any analytic function of $\mathrm{W}_{1}$ is a solution of (4). (X. L. Xobolev called such solution class functional invariant solution).

For $r<a t, c_{1}$ is imaginary, therefore a real solution has form $j=\operatorname{Re} f\left(q+c_{1}\right), r<a t$. With this, Eq. (4) become

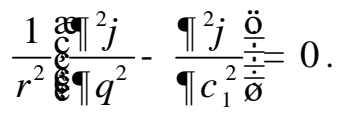

With $r^{3}$ at , $c_{1}$ is real, therefore Eq. (4) is hyperbolic.

Similarly, for $y=\operatorname{ReY}\left(q+c_{2}\right), r<b t \quad$ and $y=\mathrm{Y}\left(q+c_{2}\right), r^{3} b t$

The boundary condition at $(q= \pm a)$ is

$$
\begin{aligned}
& \frac{\mathrm{s}_{\mathrm{s}}}{j_{r}}+\frac{1}{r} y_{q}=0 \\
& \frac{1}{\mathrm{~s}} \frac{1}{r} j_{q}-y_{r}=0
\end{aligned}
$$

At the wave front, the values of $j$ and $y$ has the same limits when crossing the boundary from outside to inside. Therefore

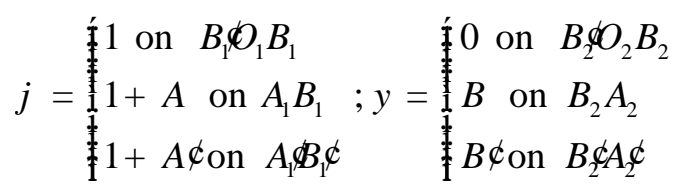

\section{Convert the Problem to the System of Integral Equation with Singularity}

Peforming the transformation $z_{1}=q+c_{1}, z_{2}=q+c_{2}$ through the variables

$$
w_{1}=\frac{1}{\sin \frac{p}{2 a} z_{1}}, w_{2}=\frac{1}{\sin \frac{p}{2 a} z_{2}}
$$

Using a conformal map, the regions $O C_{2} A_{1} B_{1} O_{1} B_{1} A_{1} C_{2} O$ and $O C_{2} A_{2} B_{2} O_{2} B_{2} A_{2} C_{2} O$ are transformed to two lower halves of the planes:

\begin{tabular}{|c|c|c|c|c|c|c|c|c|}
\hline$-¥$ & $-\frac{1}{p_{1}}$ & -1 & $-\frac{1}{k_{1}}$ & & $\frac{1}{k_{1}}$ & & & $¥$ \\
\hline$O_{1}$ & $B_{1}$ & $A_{1} \varnothing$ & $C_{2}^{\varnothing}$ & $O$ & $C_{2}$ & $A_{1}$ & $B_{1}$ & $O_{1}$ \\
\hline$-¥$ & & & -1 & & 1 & & & $¥$ \\
\hline$O_{2}$ & $B_{2}$ & $A_{2}$ & $C_{2}^{\varnothing}$ & $O$ & $C_{2}$ & $A_{2}$ & $B_{2}$ & $O_{2}$ \\
\hline
\end{tabular}

We get

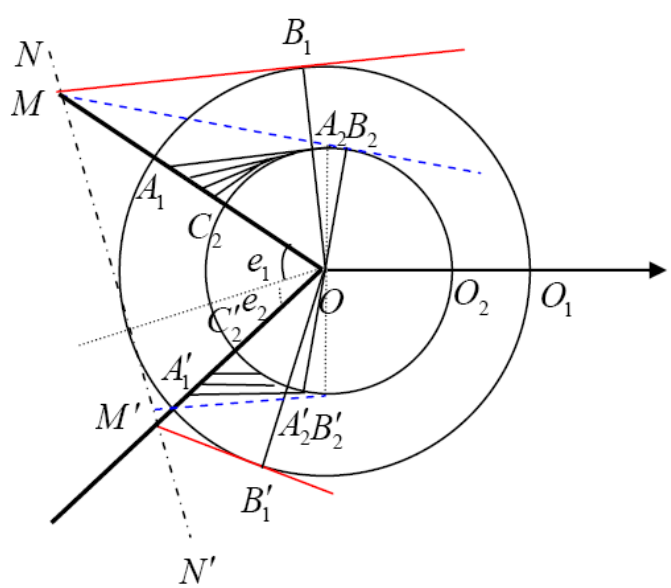

Figure 1: Shematic model of wave diffraction in elastic medium with opening angle $b=e_{1}+e_{2}$ with hard wall.

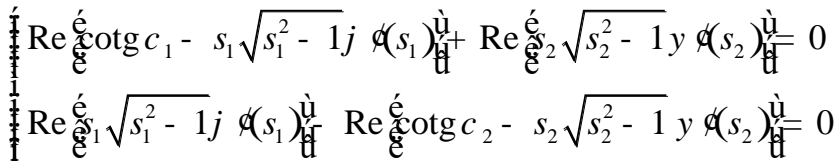

where $\frac{s_{1}}{s_{2}}=\frac{\cos \frac{p}{2 a} c_{2}}{\cos \frac{p}{2 a} c_{1}}$ và $\frac{\cos c_{2}}{\cos c_{1}}=\frac{b}{a}, \quad s_{1}, s_{2} \quad$ are

boundary values of $w_{1}, w_{2}$,

$$
s_{1}=\frac{1}{\sin \frac{p}{2 a}\left(-a+c_{1}\right)}=-\frac{1}{\cos \frac{p}{2 a} c_{1}} ; s_{2}=\frac{1}{\sin \frac{p}{2 a}\left(-a+c_{2}\right)}=-\frac{1}{\cos \frac{p}{2 a} c_{2}}
$$

From (8) we get:

$$
\frac{d w_{i}}{d z_{1}}=-\frac{p}{2 a} w_{i} \sqrt{w_{i}^{2}-1}, \quad(i=1,2)
$$

Therefore, $(*)$ becomes 


\section{International Journal of Science and Research (IJSR) \\ ISSN (Online): 2319-7064}

Index Copernicus Value (2013): 6.14 | Impact Factor (2014): 5.611

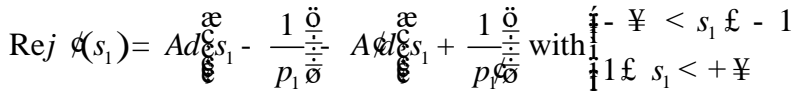

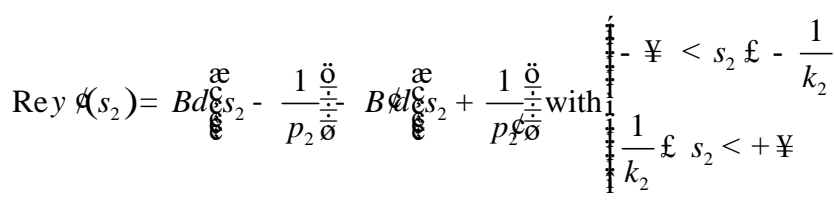

We seek the solution to Eq. (9), (10) in the form: $j \varnothing\left(w_{1}\right)=j \varnothing\left(w_{1}\right)+F_{1}\left(w_{1}\right) ;$

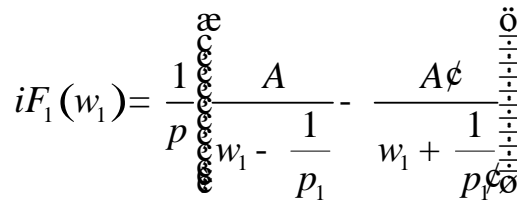

$y \not\left(w_{2}\right)=\bar{y} \not\left(w_{2}\right)+F_{2}\left(w_{2}\right)$;

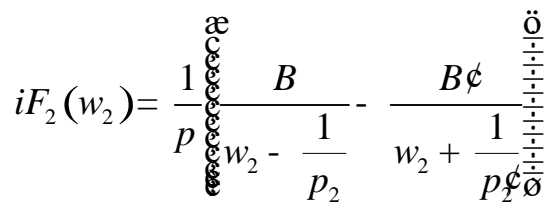

Set

$\mathrm{F}_{1}\left(w_{1}\right)=w_{1} \sqrt{w_{1}^{2}-1} j \propto\left(w_{1}\right) ; \mathrm{F}_{2}\left(w_{2}\right)=w_{2} \sqrt{w_{2}^{2}-1} \bar{y}_{1} d\left(w_{2}\right)$ one get the system of equations:

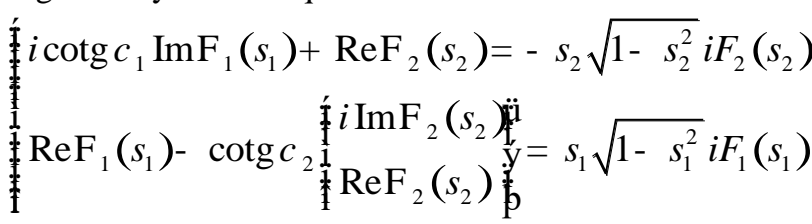

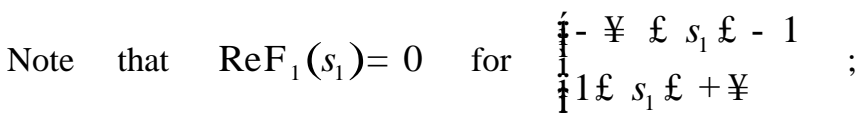

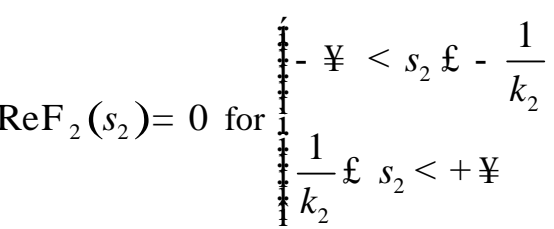

Denote $u_{2}=\operatorname{ReF}_{1}, u_{1}=\operatorname{ReF}_{2}$. One gets the following system of intergral equation with singularity:

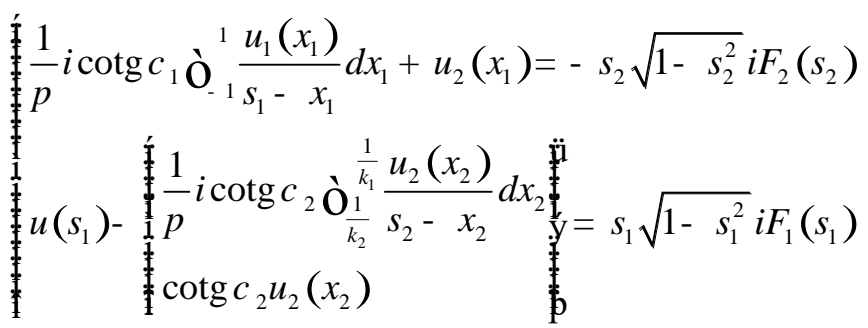

In the second equation, the upper part in the brackets is for the case $s_{2} \hat{I}[-1,1]$, and for the lower part

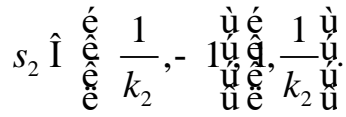

Intergral average differential (IAD) method for solving (12)

Denote $u_{2}\left(x_{2}\right)=u_{2} \dot{g}(x) \underline{\mathrm{u}}(x), s_{1}=s, s_{2}=a(s) ; \quad a(s)=\frac{\cos \frac{p}{2 a} c_{1}}{\cos \frac{p}{2 a} c_{2}} \quad$ and $\quad \frac{\cos c_{2}}{\cos c_{1}}=\frac{b}{a}, \quad$ from $\quad$ which

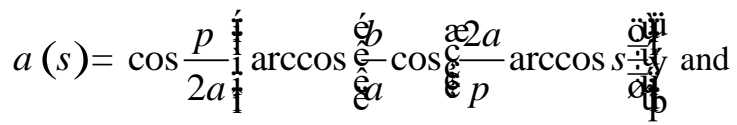

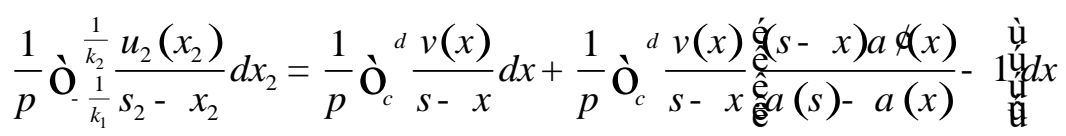

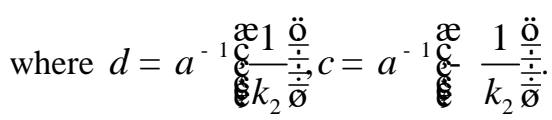

System (12) becomes:

$$
\begin{gathered}
\frac{1}{p} i \operatorname{cotg} c_{1} \mathrm{O}_{-1} \frac{1}{s-x} d x+v(s)=f_{1}(s) \\
u(s)-\frac{1}{p} i \operatorname{cotg} c_{2} \grave{\mathbf{O}}_{c} \frac{v(x)}{s-x} d x-\frac{1}{p} i \operatorname{cotg} c_{2} \grave{\mathrm{O}}_{c}{ }^{d} k(s, x) v(x) d x=f_{2}(s)
\end{gathered}
$$

with

$$
\begin{aligned}
& f_{1}(s)=-a(s) \sqrt{1-a^{2}(s)} i F_{2}(a(x)) ; f_{2}(s)=-s \sqrt{1-s^{2}} i F_{1}(s)
\end{aligned}
$$

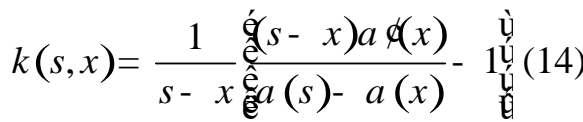




\section{International Journal of Science and Research (IJSR) \\ ISSN (Online): 2319-7064 \\ Index Copernicus Value (2013): 6.14 | Impact Factor (2014): 5.611}

One can apply directly the method for solving system of integral equation with singularity as presented in Reference [8] to solve (13). Below, we use the IAD method [5]. Set

$$
\grave{\mathrm{O}}_{-1} \frac{u(x)}{s-x} d x=S_{1} u ; \quad \dot{\mathrm{O}}_{c} \frac{d}{s-x} d x=S_{2} v
$$

From the first equation in (13) one gets $v(s)=f_{1}(s)-\frac{1}{p} i \operatorname{cotg} c_{1} S_{1} u$. Substitute into the second equation we have:

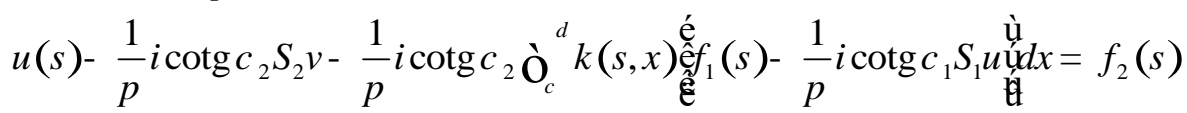

where $k(s, x)$ is given in Eq.

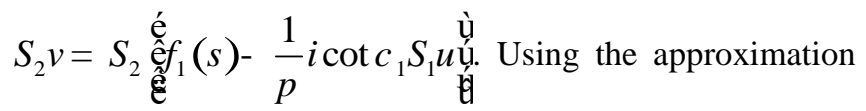
method of singularity integral of Phan Van Hap [6] for the case $b=\frac{p}{2}, b=\frac{p}{3} ; l=1, m=0,05, r=2$, we obtain the following results:

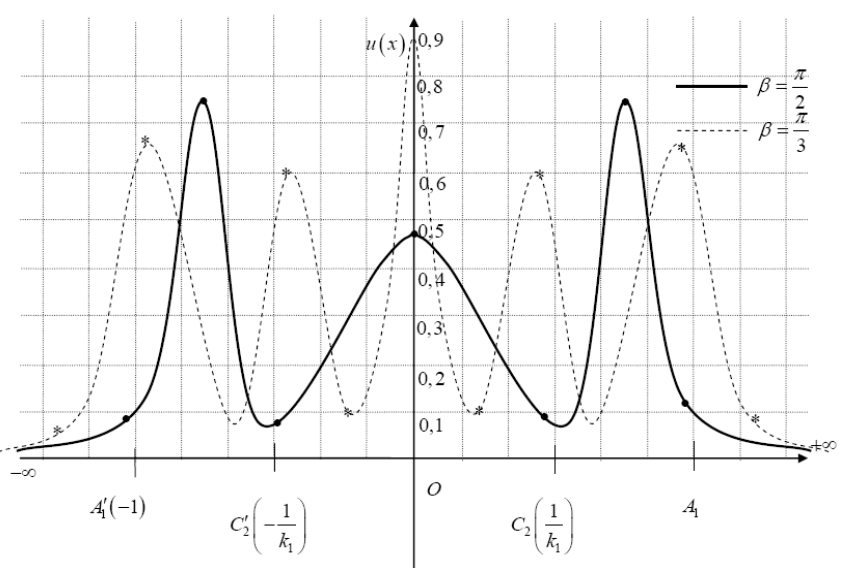

Figure 2: Diffraction function $u(x)$ calculated for the opening $b=\frac{p}{2}$ and $b=\frac{p}{3}$ as shown in Fig.1.

Compared to a recent paper [4]. We can see that our IAD method is much simpler than the method of finite difference. We also show that the equation (1.20) is simple analytically. Using this method, the obtained solutions agree well with the phenomenon of wave diffraction at the singular points considered. If one uses the method of reference [4] the numerical procedure is more complicated.

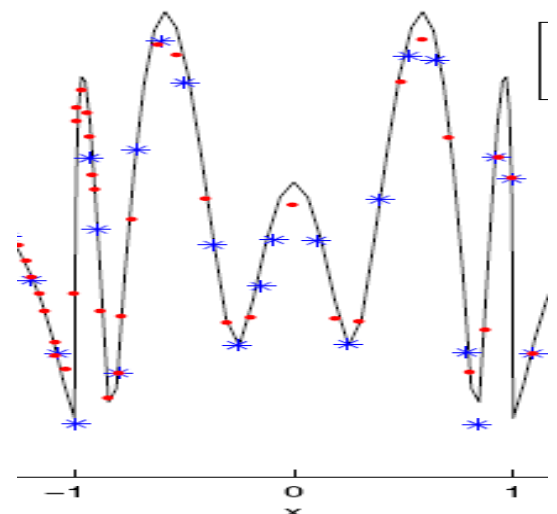

Figure 3: Comparison with results of reference [4]

\section{Conclusion}

Using the IAD method, we overcome the difficulties of working with singular points by solving the system of intergral equations with singularity using a conformal mapping of complex functions. Current theory can be expanded to study wave diffraction problems without resorting to finite difference method. The IAD method has demonstrated its advantages in solving different problems in physics and mechanics.

\section{Acknowledgement}

The authors would like to thank our colleagues, Profs . Pham ky Anh, Nguyen Suan Han, Nguyen Quang Bau, for valuable discussions and comments. This work is supported financially by the grant from the Vietnam NAFOSTED agency, grant number 103.03 - 2012.2.

\section{References}

[1] Phan Huy Thien (2015). AD method for solving the neutron transport equation in nuclear reactor", VNU Journal of Mathematics - Physics. (ISSN 0866-8612). 31(1S), pp. 153-160.

[2] Hoang Anh Tuan, Phan Huy Thien, Phan Van Hap. Advanced precision approximate solution of equations by means of continuous parameters. Proceedings of International Conference on Physics of nuclear reactor 19 th. Budapest, Hungary, 193 - 201, 1990.

[3] Фан-Ван-Хап (1976) О некоторых итерационных методах приближенного решения операторных уравнений и их применении, Изв. вузов. Матем., № 4, 87-94.

[4] Z J Fu, W Chen, J T Chen and W Z Qu, Singular boundary method: three regularization approaches and exterior wave applications, CMES, 2014, Vol.99, No.5, pp.417- 443.

[5] Фан-Ван-Хап. Вестник МГУ. 1966, 5, 41-59.

[6] Фан-Ван-Хап. Дубна Р.5, 3642,1967.

[7] Фан-Ван-Хап. ЖВМ. МФ. 1965, 5, 2. 171-184.

[8] Phan Van Hap, Acta Sientiarum Vietnamicarum, T VI, 1970.

[9] J. J. Golecki (2007). Direct diplacement method in crack theory (numerical resolution), Mecanica. 42: 555566.

[10] Phan Van Hap, Phan Huy Thien (1994), The AD method for the approximate solution of the Wigner equation Demonstratio Mathematica Vol. XXVII, No 2, pp. 283286. 